\title{
Ann Arbor Stage IE
}

National Cancer Institute

\section{Source}

National Cancer Institute. Ann Arbor Stage IE. NCI Thesaurus. Code C150547.

An Ann Arbor classification lymphoma stage term that refers to the involvement of a single extranodal organ or site in the absence of any lymph node involvement. 